\title{
Análisis coste-efectividad y coste-utilidad de apixaban frente a dabigatrán y rivaroxaban en el tratamiento y prevención secundaria del tromboembolismo venoso
}

\author{
Fernando de Andrés-Nogales ${ }^{1}$ - Itziar Oyagüez ${ }^{1}$ Luis Antonio Álvarez-Sala ${ }^{2}$. \\ Fernando García-Bragado $^{3}$ • Andrés Navarro ${ }^{4}$ - Paloma González ${ }^{5}$. Isabel Elías ${ }^{1}$. \\ Javier Soto ${ }^{6}$
}

Published online: 26 January 2017

(c) The Author(s) 2017. This article is published with open access at Springerlink.com

Resumen Objetivo Estimar la relación coste-utilidad de seis meses de tratamiento con apixaban $(10 \mathrm{mg} / 12 \mathrm{~h}$, siete primeros días; $5 \mathrm{mg} / 12 \mathrm{~h}$ después) para el primer evento de tromboembolismo venoso (TEV) y prevención de recurrencias frente a dabigatrán $(150 \mathrm{mg} / 12 \mathrm{~h})$ después de cinco días con heparina de bajo peso molecular (HBPM/dabigatrán) y frente a rivaroxaban $(15 \mathrm{mg} / 12 \mathrm{~h}$ los 21 primeros días; después $20 \mathrm{mg} / \mathrm{día}$ ).

Material y métodos Se simuló con un modelo de Markov la evolución de la enfermedad en 1.000 pacientes adultos a lo largo de su vida. La eficacia se obtuvo de un metanálisis, calculándose los años de vida ganados (AVG) y años de vida ajustados por calidad (AVAC) obtenidos con las opciones terapéuticas evaluadas. Según la perspectiva del Sistema Nacional de Salud (SNS) se incluyeron los costes sanitarios directos. Los costes unitarios ( $€$ de 2014) fueron obtenidos de la literatura y bases de datos nacionales. Se aplicó una tasa de descuento $(3 \%)$ a costes y beneficios en salud.

$\triangle$ F. de Andrés-Nogales

fdeandres@porib.com

1 Pharmacoeconomics \& Outcomes Research Iberia (PORIB), Paseo Joaquín Rodrigo, 4 letra I, 28224 Pozuelo de Alarcón, Madrid, España

2 Servicio de Medicina Interna, Hospital General Universitario Gregorio Marañón, Facultad de Medicina, Universidad Complutense de Madrid, Madrid, España

3 Servicio de Medicina Interna, Hospital Universitari de Girona Doctor Josep Trueta, Gerona, España

4 Servicio de Farmacia, Hospital General Universitario de Elche, Alicante, España

5 Departamento Health Economics, Bristol-Myers Squibb, Madrid, España

6 Departamento Health Economics, Pfizer, Alcobendas, Madrid, España
Se realizaron análisis de sensibilidad (AS) determinísticos y probabilístico.

Resultados Al finalizar la simulación apixaban resultó una terapia más efectiva, 7,182 AVG y 5,865 AVAC, que HBPM/dabigatrán (7,162 AVG y 5,846 AVAC) y con menor coste total $(13.374,70 €$ frente a $13.516,50 €)$. Igualmente frente a rivaroxaban $(7,174 \mathrm{AVG}$ y $5,857 \mathrm{AVAC}$ y coste total $13.537,8 €$ ). El AS probabilístico confirmó el dominio de apixaban.

Conclusiones Apixaban fue una alternativa dominante frente a HBPM/dabigatrán y frente a rivaroxaban para el SNS en el tratamiento de TEV y prevención de sus recurrencias.

Palabras clave Apixaban - Anticoagulante ·

Coste-utilidad · Embolia pulmonar · Tromboembolismo venoso $\cdot$ Trombosis venosa profunda

Abstract Objective Cost-utility analysis of a 6-month treatment of apixaban $(10 \mathrm{mg} / 12 \mathrm{~h}$, first 7 days; $5 \mathrm{mg} / 12 \mathrm{~h}$, afterwards) for the treatment of the first event of venous thromboembolism (VTE) and prevention of recurrences, versus dabigatran $(150 \mathrm{mg} / 12 \mathrm{~h})$ after 5 days taking low molecular weight heparins (LMWH/dabigatran) and versus rivaroxaban $(15 \mathrm{mg} / 12 \mathrm{~h}$, the first 21 days; $20 \mathrm{mg} /$ day, afterwards). Material and methods The course of the disease of a 1,000 adult patients was modeled using a life-long Markov model. Efficacy was obtained from a meta-analysis, calculating life years gained (LYG) and quality adjusted life years (QALYs) obtained from the therapeutic options evaluated. Based on a Spanish National Health System (NHS) perspective, only direct medical costs were included. Unit costs $(€, 2014)$ were obtained from literature review and national databases. A 3\% discount rate was applied for health outcomes and costs. Several sensibility analyses (SA) were performed. Results Apixaban resulted the most effective therapy with 7.182 LYG and 5.865 QALYs, versus 7.162 LYG and 
5.846 QALYs with LMWH/dabigatran and lower total cost $(€ 13,374.70$ vs $€ 13,516.50)$. LYG and QALYs with rivaroxaban were 7.174 and 5.857 and a total cost of $€ 13,537.80$. Probabilistic SA confirmed dominance of apixaban versus LMWH/dabigatran and rivaroxaban.

Conclusions Apixaban versus LMWH/dabigatran and versus rivaroxaban was a dominant treatment for the NHS perspective for VTE treatment and prevention of recurrences.

Keywords Apixaban - Anticoagulants · Cost-utility · Pulmonary embolism $\cdot$ Venous thromboembolism $\cdot$ Deep vein thrombosis

\section{Introducción}

La enfermedad tromboembólica venosa o tromboembolismo venoso (TEV) es un proceso grave y potencialmente mortal, caracterizado por la aparición de un trombo formado, inicialmente, por plaquetas y fibrina en el interior del sistema venoso profundo, que puede crecer y fragmentarse. En este último caso, uno de los fragmentos puede desprenderse, progresar en la dirección del flujo sanguíneo y llegar al pulmón provocando una embolia pulmonar (EP) [1]. Por todo ello, actualmente se considera que la trombosis venosa profunda (TVP) y la EP son dos manifestaciones de la misma enfermedad, a la que llamamos TEV y que constituye la tercera causa de muerte cardiovascular a nivel global, tras la cardiopatía isquémica y el ictus [1,2].

Con alrededor de un $60 \%$ de TVP y un $40 \%$ de EP $[3,4]$ la prevalencia total de TEV se estimó en España en el $0,11 \%$ [5], con un aumento anual progresivo de los casos alcanzando una tasa de diagnóstico de hasta 154/100.000 habitantes, incluyendo los diagnósticos no hospitalarios [6].

Los pacientes con TEV tienen un riesgo elevado de presentar recurrencias, siendo este riesgo mayor en los dos primeros años [7]. Además, pueden desarrollar complicaciones a largo plazo como el síndrome postrombótico (SPT) y la hipertensión pulmonar crónica (HPTEC) [8].

El TEV genera un importante consumo de recursos debido a estas complicaciones y a las recurrencias que requieren hospitalización. El coste total asociado al TEV en España se estimó en más de 75,5 millones de euros en el año 2005 , con el 79,3\% del coste correspondiente a la asistencia sanitaria hospitalaria, e incrementándose a un ritmo del 8-9\% anual [9]. El manejo terapéutico fundamental del TEV es la anticoagulación, con intervenciones como trombólisis y filtros de vena cava inferior reservadas para situaciones especiales [8]. Las guías actuales recomiendan el tratamiento en fase aguda con anticoagulación parenteral durante 510 días, seguida del tratamiento con anticoagulantes orales como antagonistas de la vitamina $\mathrm{K}(\mathrm{AVK})$, dabigatrán o edoxabán, durante un tratamiento mínimo de tres meses.
Otros fármacos anticoagulantes orales, como apixaban o rivaroxaban no requieren tratamiento parenteral inicial, sustituyéndose por una mayor dosis de anticoagulante oral durante los primeros días [10].

Se realizó un análisis coste-efectividad y coste-utilidad con el objetivo de evaluar las implicaciones clínicas y económicas del tratamiento del primer evento de TEV y prevención de sus recurrencias con apixaban en comparación con el tratamiento con rivaroxaban y con heparinas de bajo peso molecular (HBPM) y dabigatrán.

\section{Material y métodos}

Se estimaron los costes y beneficios en salud de una cohorte de 1.000 pacientes que requerían tratamiento con anticoagulantes para el tratamiento de un primer evento de TEV y de sus recurrencias en España, empleándose para ello un modelo analítico de decisión.

\section{Estructura del modelo}

Para el análisis se utilizó un modelo de Markov, una técnica ampliamente utilizada en evaluaciones económicas de tecnologías sanitarias $[11,12]$. La estructura del modelo, con 13 estados de salud y equivalente al modelo empleado en una evaluación previa de la eficiencia de apixaban frente a HBPM/AVK [13], se diseñó en función de una revisión de la literatura disponible y de los modelos previamente aceptados por el National Institute for Care and Health Excellence (NICE) para esta patología [14, 15]. La Fig. 1 muestra el diagrama en el que se representan dichos estados de salud junto con las posibles transiciones entre ellos. Los pacientes se sitúan inicialmente en los estados de salud "primer evento de EP o TVP", realizándose transiciones entre los distintos estados de salud en ciclos de tres meses de duración, pudiéndose experimentar solo un evento en cada ciclo. El modelo asume que después de haber experimentado un evento no existe ningún impacto en el riesgo de eventos sucesivos ni en el tratamiento anticoagulante posterior. Además, se consideró la tasa de mortalidad por cualquier causa diferenciando por género y edad.

\section{Alternativas terapéuticas}

Las tres alternativas a comparar en este estudio fueron HBPM (enoxaparina)/dabigatrán y rivaroxaban frente a apixaban. Las posologías consideradas fueron $150 \mathrm{mg} / 12 \mathrm{~h}$ con dabigatrán tras cinco días con enoxaparina $(1 \mathrm{mg} / \mathrm{kg})$. Con rivaroxaban, $15 \mathrm{mg} / 12 \mathrm{~h}$ durante los primeros 21 días y $20 \mathrm{mg} /$ día tras ese periodo. Con apixaban, la dosis fue $10 \mathrm{mg} / 12 \mathrm{~h}$ durante los siete primeros días, continuando con $5 \mathrm{mg} / 12 \mathrm{~h}$. La duración del tratamiento considerada fue seis meses en todos los casos. 
Tabla 1 Eficacia de las alternativas consideradas

\section{FASE AGUDA $^{\mathrm{a}}$}

RR del TEV recurrente y muerte relacionada con el TEV (IC 95\%)

Dabigatrán

Rivaroxaban

$1,31(0,79-2,16)$

$1,08(0,69-1,69)$

Distribución del TEV recurrente y muerte relacionada con TEV

\begin{tabular}{|c|c|c|c|c|c|}
\hline \multicolumn{2}{|c|}{ Muerte relacionada con TEV } & \multicolumn{2}{|c|}{ EP no fatal recurrente } & \multicolumn{2}{|c|}{ TVP no fatal recurrente } \\
\hline$\overline{\mathrm{N}}$ & $\%$ & $\overline{\mathrm{N}}$ & $\%$ & $\overline{\mathrm{N}}$ & $\%$ \\
\hline 28 & $21,54 \%$ & 49 & $37,69 \%$ & 53 & $40,77 \%$ \\
\hline
\end{tabular}

RR hemorragias graves (IC 95\%)

Dabigatrán Rivaroxaban

$2,51(1,23-5,34)$

$1,83(0,92-3,76)$

RR de sangrado NMCR (IC 95\%)

\begin{tabular}{ll}
\hline Dabigatrán & Rivaroxaban \\
\hline $1,25(0,89-1,75)$ & $2,12(1,62-2,80)$
\end{tabular}

RR tras la discontinuación del tratamiento (independiente de los eventos clínicos incluidos en el modelo) (IC 95\%)

Dabigatrán Rivaroxaban

$1,17(0,97-1,41) \quad 0,91(0,77-1,09)$

\section{PERIODO POSTERIOR}

RR del TEV recurrente y muerte relacionada con el TEV (IC 95\%)

\begin{tabular}{lllll}
\hline Dabigatrán & & & Rivaroxaban \\
\hline Frente a 2,5 mg & Frente a 5 mg & & Frente a 2,5 mg & Frente a 5 mg \\
\hline $0,56(0,21-1,44)$ & $0,55(0,21-1,40)$ & $0,99(0,37-2,52)$ & $0,96(0,35-2,43)$
\end{tabular}

Distribución del TEV recurrente y muerte relacionada con TEV

\begin{tabular}{|c|c|c|c|c|c|}
\hline & TEV & EP & & $\mathrm{TV}$ & \\
\hline$\overline{\mathrm{N}}$ & $\%$ & $\overline{\mathrm{N}}$ & $\%$ & $\overline{\mathrm{N}}$ & $\%$ \\
\hline 12 & $11,88 \%$ & 25 & $24,75 \%$ & 64 & $63,37 \%$ \\
\hline
\end{tabular}

RR hemorragias graves (IC 95\%)

\begin{tabular}{|c|c|c|c|}
\hline \multicolumn{2}{|l|}{ Dabigatrán } & \multicolumn{2}{|l|}{ Rivaroxaban } \\
\hline Frente a $2,5 \mathrm{mg}$ & Frente a $5 \mathrm{mg}$ & Frente a $2,5 \mathrm{mg}$ & Frente a $5 \mathrm{mg}$ \\
\hline$\underline{4,21(0,55-44,26)}$ & $9,96(0,91-329)$ & $39,75(1,54-1086)$ & $100(2,78-6305)$ \\
\hline \multicolumn{4}{|c|}{ RR de sangrado NMCR (IC 95\%) } \\
\hline Dabigatrán & & Rivaroxaban & \\
\hline Frente a $2,5 \mathrm{mg}$ & Frente a $5 \mathrm{mg}$ & Frente a $2,5 \mathrm{mg}$ & Frente a $5 \mathrm{mg}$ \\
\hline $2,13(0,89-5,13)$ & $1,50(0,64-3,51)$ & $3,58(1,36-9,69)$ & $2,51(0,98-6,67)$ \\
\hline
\end{tabular}

RR tras la discontinuación del tratamiento (independiente de los eventos clínicos incluidos en el modelo) (IC 95\%)

\begin{tabular}{lllll}
\hline Dabigatrán & \multicolumn{2}{c}{ Rivaroxaban } \\
\hline Frente a 2,5 mg & Frente a 5 mg & & Frente a 2,5 mg & Frente a 5 mg \\
\hline $1,27(0,92-1,76)$ & $1,09(0,79-1,49)$ & $1,38(0,97-1,97)$ & $1,18(0,83-1,66)$ \\
\hline
\end{tabular}

aDatos del metanálisis fase aguda

${ }^{\mathrm{b}}$ Datos del metanálisis periodo posterior

EP: embolia pulmonar; NMCR: no mayor clínicamente relevante; RR: riesgo relativo; TEV: tromboembolismo venoso; TVP: trombosis venosa profunda 


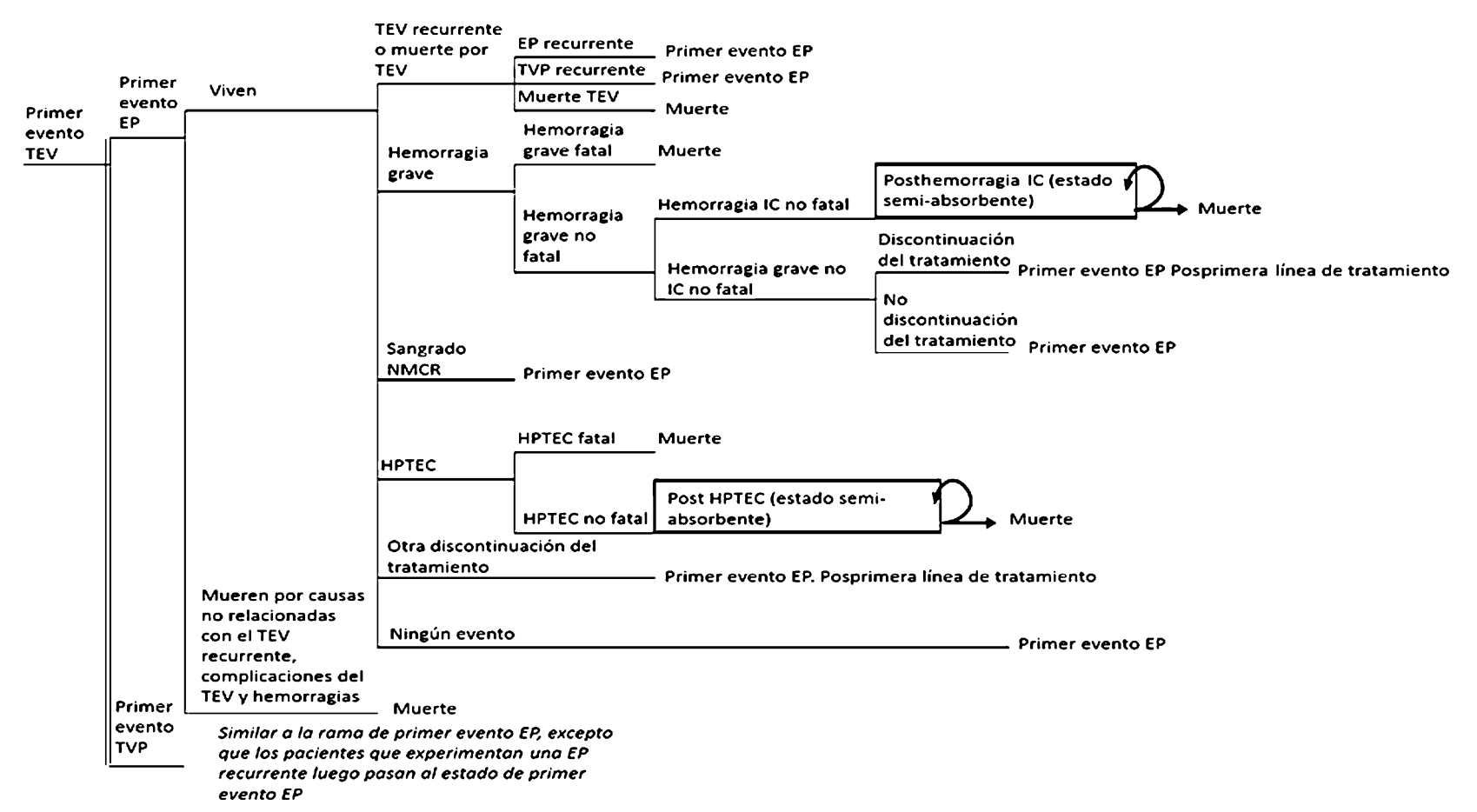

Figura 1 Modelo de Markov. EP: embolia pulmonar; HPTEC: hipertensión pulmonar tromboembólica crónica; IC: intracraneal; NMCR: no mayor clínicamente relevante; TEV: tromboembolismo venoso; TVP: trombosis venosa profunda

\section{Perspectiva, tasa de descuento y horizonte temporal}

En el análisis se consideró la perspectiva del Sistema Nacional de Salud (SNS), aplicándose una tasa de descuento del $3 \%$ anual a los costes y a los beneficios en salud durante un horizonte temporal correspondiente a toda la vida del paciente.

\section{Resultados en salud}

En el presente modelo se refleja el riesgo de desarrollar cada uno de los siguientes eventos clínicos: TEV recurrente y muerte relacionada con el TEV, hemorragias graves, sangrado no mayor clínicamente relevante (NMCR), SPT y HPTEC (Tabla 1). El riesgo de desarrollar algún evento clínico se desglosa en dos periodos; una fase aguda inicial de seis meses y un periodo posterior hasta alcanzar el horizonte temporal considerado en el análisis, toda la vida del paciente. Dado que no se han realizado ensayos clínicos en los que se hayan comparado de manera directa las alternativas en estudio (apixaban, dabigatrán y rivaroxaban), los datos de resultados en salud incorporados en el modelo se han obtenido de comparaciones indirectas y metanálisis en red realizadas entre las tres alternativas comparadas [16, 17], lo que va a permitir establecer la eficacia relativa entre los tres medicamentos evaluados en esta evaluación económica.

El riesgo de padecer un evento clínico tras discontinuar el tratamiento permanentemente (por discontinuación o tras completar el tratamiento) se obtuvo de la literatura [18, 19] y de la rama del estudio pivotal de extensión que comparó apixaban con HBPM/AVK (AMPLIFY-EXT), en la que se utilizó el placebo como comparador [20]. La frecuencia de HPTEC $(1,25 \%)$ [21] y la prevalencia del SPT $(8,1 \%)$ [22], que se consideró constante, fueron obtenidas de la literatura.

El tratamiento anticoagulante recibido no afectó a la naturaleza de las hemorragias fatales, considerando que el $13,46 \%$ de todas las hemorragias graves fueron fatales y, de las no fatales restantes, el 13,97\% fueron intracraneales [23]. Además, a partir de los 18 meses tras el evento embólico se aplicó un factor incremental de riesgo de sangrado de 0,197 por década vivida, dado que la edad es un factor adicional de riesgo de sangrado [24]. En relación con la discontinuación del tratamiento por eventos hemorrágicos, según datos de un análisis secundario del estudio pivotal de apixaban frente a HBPM/AVK (AMPLIFY) [25] validados por el panel de expertos, se consideró una discontinuación de dos días en sangrado NMCR y permanente en el caso de hemorragias intracraneales. En hemorragia grave no intracraneal el 47,27\% de los pacientes discontinuó durante 14 días, mientras que el resto discontinuó permanentemente.

\section{Medición del valor de utilidad asociado con los fármacos en evaluación}

Para la estimación de los años de vida ajustados por calidad (AVAC), los cuales valoran los años de vida ganados ajustados por la utilidad de los pacientes (grado de preferencia 
Tabla 2 Disutilidades por evento clínico, sangrados y tratamiento anticoagulante

\begin{tabular}{lll}
\hline Valor disutilidad & Fuente disutilidad & Duración \\
\hline Eventos clínicos & & \\
$\quad$ EP & $-0,32[27]$ & 30 días [29, 30] \\
TVP & $-0,11[27]$ & 30 días [29, 30] \\
Sangrados & & \\
$\quad$ Sangrado no IC & $-0,30[27]$ & 30 días [29, 30] \\
$\quad$ Sangrado NMCR & $-0,0054[31]$ & Dos días [Opinión de los expertos] \\
SPT grave & $-0,0700[32]$ & Durante el SPT [32] \\
Anticoagulantes & & \\
$\quad \begin{array}{l}\text { Apixaban } \\
\text { HBPM }\end{array}$ & $-0,002[33]$ & Durante el tratamiento [33] \\
Dabigatrán & $-0,013[33]$ & Durante el tratamiento [33] \\
Rivaroxaban & $-0,002[33]$ & Durante el tratamiento [33] \\
\end{tabular}

de los pacientes por su estado de salud), se consideró la influencia que tiene la terapia anticoagulante sobre el grado de utilidad en los pacientes con TEV. El modelo consideró diferentes valores de utilidad en función del estado de salud de los pacientes. Como valor de utilidad basal, para reflejar el nivel de calidad de vida relacionada con la salud (CVRS) de un paciente con TEV sin historial de complicaciones, se asumió un valor de 0,825 [26]. La aparición de una hemorragia intracraneal o HPTEC se asoció con valores de utilidad de 0,330 [27] y 0,650 [28], respectivamente.

Se consideraron también decrementos de utilidad asociados al desarrollo de eventos clínicos y sangrados, así como a la vía de administración del tratamiento anticoagulante, con un mayor impacto de la vía parenteral en la CVRS (Tabla 2) [27, 29-33].

\section{Recursos consumidos y costes asociados}

En línea con la perspectiva del análisis, únicamente se incluyeron costes sanitarios directos: coste farmacológico, coste de administración, coste de monitorización del cociente internacional normalizado y coste de manejo del TEV y de sus complicaciones. El coste de los tratamientos farmacológicos se calculó a partir del precio de venta al público recogido en el Catálogo de medicamentos del Consejo General de Colegios Oficiales de Farmacéuticos [34], aplicando las deducciones vigentes correspondientes al Real DecretoLey 8/2010 [35]. La identificación y estimación de los recursos necesarios para la monitorización del tratamiento y el manejo de la enfermedad y de las complicaciones (medicación, número de consultas necesarias, pruebas diagnósticas, etc.) fue llevada a cabo por el panel de expertos incorporado a este análisis. Los costes unitarios de cada recurso fueron obtenidos de la literatura y de una base de datos nacional de costes sanitarios, aplicándose en los casos necesarios la variación del índice de precios de consumo correspondiente desde el año de origen de los costes localizados al año del presente análisis [36-41]. Todos los costes incluidos en el modelo se expresaron en euros de 2014 y aparecen detallados en la Tabla 3.

\section{Análisis de los resultados en salud obtenidos y costes producidos}

La eficacia de las alternativas evaluadas condicionó la aparición de eventos clínicos y, consecuentemente, la supervivencia final de la cohorte analizada. Para analizar la eficiencia se consideraron tanto los costes totales asociados a cada una de las alternativas como los resultados en salud en términos de supervivencia media por paciente, medidos como años de vida ganados (AVG) al final de la simulación, y que posteriormente fueron ajustados con los valores de utilidad correspondientes para expresarse como AVAC acumulados al final de todos los ciclos del modelo. La estimación de la relación coste-efectividad incremental (RCEI) y costeutilidad incremental (RCUI) entre las alternativas del análisis se realizó mediante las siguientes fórmulas:

$R C E I=\frac{\text { Coste }_{\text {apixaban }}-\text { Coste }_{\text {comparador }}}{A V G_{\text {apixaban }}-A V G_{\text {comparador }}}$
$R C U I=\frac{\text { Coste }_{\text {apixaban }}-\text { Coste }_{\text {comparador }}}{A V A C_{\text {apixaban }}-A V A C_{\text {comparador }}}$

\section{Análisis de sensibilidad}

Se llevaron a cabo tanto análisis de sensibilidad determinísticos (ASD) univariantes como probabilístico (ASP), con el fin de evaluar la repercusión de la variabilidad de determinados parámetros sobre los resultados del modelo y confirmar así su robustez. En los ASD se modificaron de forma individual distintos parámetros: duración del tratamiento (tres, 
Tabla 3 Costes unitarios (€ de 2014)

\section{COSTE FARMACOLÓGICO}

\begin{tabular}{|c|c|c|c|}
\hline Principio activo & Dosis & $\begin{array}{l}\text { Coste tratamiento diario durante } \\
\text { el periodo de inducción }(€)\end{array}$ & $\begin{array}{l}\text { Coste tratamiento diario } \\
\text { hasta completar seis } \\
\text { meses }(€)\end{array}$ \\
\hline Apixaban & $\begin{array}{l}10 \text { mg b.i.d. (siete primeros días) } \\
5 \text { mg b.i.d. (hasta completar los } \\
\text { seis meses) }\end{array}$ & 5,60 & 2,80 \\
\hline HBPM & $1 \mathrm{mg} / \mathrm{kg} / \mathrm{b} . i . d$. (5 primeros días) & 15,66 & Dabigatrán \\
\hline Dabigatrán & $\begin{array}{l}150 \text { mg b.i.d. (hasta completar los } \\
\text { seis meses) }\end{array}$ & HBPM & 2,80 \\
\hline Rivaroxaban & $\begin{array}{l}15 \mathrm{mg} \text { b.i.d ( } 21 \text { primeros días) } \\
20 \mathrm{mg} / \text { día (hasta completar los } \\
\text { seis meses) }\end{array}$ & 5,60 & 2,80 \\
\hline
\end{tabular}

\section{COSTE DE ADMINISTRACIÓN}

\begin{tabular}{ll}
\hline Concepto & Coste unitario (€) \\
\hline Educación de un paciente & 17,94 \\
Administración de HBPM en el centro de salud & 14,53 \\
\hline
\end{tabular}

\section{COSTE DE MONITORIZACIÓN INR}

\section{Concepto}

Prueba de protrombina INR

Consulta médico de atención primaria

Primera consulta hematólogo

Consultas sucesivas hematólogo

\section{Coste unitario (€)}

4,71

42,88

109,49

65,69

\section{COSTE DEL MANEJO DEL TEV}

\begin{tabular}{ll}
\hline Ámbito hospitalario & Coste unitario/día $(€)$ \\
\hline Estancia en la planta de medicina interna & 502,30 \\
Estancia en la planta de neumología & 501,59 \\
\hline & \\
\hline COSTE DEL MANEJO DEL TEV & Coste unitario (€) \\
\hline Ámbito de atención primaria & 60,91 \\
\hline Ultrasonidos doppler & 212,67 \\
Angiotomografía computarizada & 97,13 \\
Ecocardiograma & 22,57 \\
Rayos-X tórax & 15,99 \\
Test-dímero D & 133,40 \\
Visita a urgencias & 207,55 \\
Primera visita medicina interna & 124,53 \\
Visita sucesiva medicina interna & \\
\hline
\end{tabular}


Tabla 3 (Continuación)

\begin{tabular}{ll}
\hline COSTE DEL MANEJO DE LAS COMPLICACIONES & \\
\hline COSTES AGUDOS $(€)$ & \\
\hline Estados de salud semiabsorbentes & $7.748,42$ \\
$\quad$ Hemorragia IC & $5.836,07$ \\
HPTEC & \\
Estados de salud transitorios $(€)$ & $3.538,30$ \\
Sangrado EC & $2.376,10$ \\
Sangrado NMCR & \\
Eventos fatales & $3.584,79$ \\
Hemorragia grave fatal & $5.836,07$ \\
HPTEC & $3.414,43$ \\
Muerte relacionada con TEV &
\end{tabular}

COSTES MANTENIMIENTO PRIMER CICLO (€)

\begin{tabular}{ll}
\hline Hemorragia IC & $1.937,10$ \\
HPTEC & $5.836,07$
\end{tabular}

COSTES MANTENIMIENTO CICLOS SUCESIVOS (€)

Hemorragia IC

HPTEC

SPT
$1.937,10$

$5.836,07$

$4.534,00$

b.i.d.: dos veces al día; EC: extracraneal; HBPM: heparinas de bajo peso molecular; HPTEC: hipertensión pulmonar tromboembólica crónica; IC: intracraneal; INR: cociente internacional normalizado (International Normalized Ratio); NMCR: no mayor clínicamente relevante; SPT: síndrome postrombótico; TEV: tromboembolismo venoso

12 meses y toda la vida del paciente), horizonte temporal (uno, cinco y 10 años) y tasa de descuento (0-5\%).

El ASP consistió en la variación simultánea (2.000 simulaciones de Montecarlo) de todos los parámetros considerados potencialmente relevantes en cuanto a su impacto en los resultados según una distribución asignada y ajustada al tipo de variabilidad que presentan. Las funciones aplicadas a los distintos parámetros fueron beta (tasa de eventos y utilidades), log-normal (costes y riesgos relativos), gamma (tasas de eventos y costes) y Dirichlet (distribución de TEV recurrente según el porcentaje de muerte relacionada con TEV, porcentaje de EP no fatal y TVP). Los parámetros de las distribuciones fueron ajustados en función del error estándar y de los intervalos de confianza de cada parámetro incluido en el análisis.

\section{Resultados}

Los resultados se presentan para una cohorte de 1.000 pacientes y un horizonte temporal del modelo correspondiente a toda la vida del paciente. Los resultados incluyeron el número de eventos TEV recurrentes, eventos hemorrágicos graves, sangrados NMCR, HPTEC, SPT y la discontinuación del tratamiento. Para el cálculo del RCEI y RCUI fueron utilizados los costes incrementales y AVG o AVAC incrementales, respectivamente. El número de eventos, los AVG y AVAC y los costes asociados a la terapia anticoagulante y al manejo de cada uno de los eventos clínicos se desglosan en la Tabla 4.

Considerando el número de eventos clínicos se observa una reducción de eventos de TEV recurrentes, hemorragias, sangrados y costes en los pacientes con TEV tratados con apixaban con respecto a los tratados con HBPM/dabigatrán y rivaroxaban. En los pacientes tratados con apixaban el número de AVG fue 7,182, mientras que en los pacientes tratados con HBPM/dabigatrán y rivaroxaban, fueron 7,162 y 7,174 , respectivamente. Según el valor de utilidad correspondiente al estado de salud de los pacientes, el promedio de AVAC ganados en pacientes tratados con apixaban fue 5,865, siendo de 5,846 y 5,857 para el tratamiento con HBPM/dabigatrán y rivaroxaban, respectivamente. El coste total por paciente correspondiente al tratamiento con apixaban fue $13.374,70 €, 13.516,50 €$ con HBPM/dabigatrán 
Tabla 4 Resultados del análisis coste-efectividad y coste-utilidad

\section{Apixaban}

HBPM/Dabigatrán

Rivaroxaban

\section{NÚMERO DE EVENTOS CLÍNICOS}

TEV recurrente y muerte relacionada con el TEV

Muerte relacionada con el TEV

EP no fatal recurrente

TVP no fatal recurrente

Total

Hemorragias graves

Fatal

IC no fatales

No IC no fatales

Total

Sangrado NMCR

Sangrado NMCR

HPTEC

HPTEC

Otra discontinuación del tratamiento

Relacionada con el evento (hemorragia grave no IC no fatal)

Otra discontinuación del tratamiento

Total

Muerte

Relacionada con el evento (agudo)

Relacionada con el evento a largo plazo (muerte debida a los estados de salud absorbentes)

No relacionadas con TEV recurrente, con sus complicaciones y con las hemorragias

Total

Primer evento embólico

Costes relacionados con el tratamiento anticoagulante

Farmacológico

Administración

Monitorización

Coste total

$188,00 €(1,41 \%)$
52

107

53

109

263

426

10

9

54

73

300

333

21

21

$(17,23 \%)$

$2.329,20 €$

$(17,21 \%)$

$543,20 €(4,06 \%)$

$0 €$

$\mathbf{7 3 1 , 2 0} €(5,47 \%)$

$597,70 €(4,42 \%)$

$581,90 €(4,30 \%)$

$11,90 €(0,09 \%)$

$0 €$

$187,70 €(1,39 \%)$

$187,80 €(1,39 \%)$

$797,30 €(5,90 \%)$

$\mathbf{7 6 9 , 7 0} €(5,69 \%)$

$157,60 €(1,18 \%)$

$322,90 €(2,41 \%)$

$589,00 €(4,40 \%)$

$162,00 €(1,20 \%)$

$158,60 €(1,17 \%)$

$330,40 €(2,44 \%)$

$324,60 €(2,39 \%)$

$593,90 €(4,39 \%)$

$589,90 €(4,36 \%)$

$1.069,50 €(7,80 \%)$ 
Tabla 4 (Continuación)

\begin{tabular}{|c|c|c|c|}
\hline & Apixaban & HBPM/Dabigatrán & Rivaroxaban \\
\hline \multicolumn{4}{|l|}{ Hemorragias graves } \\
\hline Hemorragia fatal & $25,70 €(0,19 \%)$ & $29,60 €(0,22 \%)$ & $27,90 €(0,21 \%)$ \\
\hline \multicolumn{4}{|l|}{ Hemorragia IC no fatal } \\
\hline Costes relacionados con el evento agudo & $49,90 €(0,37 \%)$ & $57,50 €(0,43 \%)$ & $54,10 €(0,40 \%)$ \\
\hline Costes relacionados con el cuidado prolongado & $149,40 €(1,12 \%)$ & $183,10 €(1,35 \%)$ & $167,90 €(1,24 \%)$ \\
\hline Hemorragia grave no IC no fatal & $140,50 €(1,10 \%)$ & $161,60 €(1,20 \%)$ & $152,10 €(1,12 \%)$ \\
\hline Coste total & $365,50 €(2,73 \%)$ & $431,70 €(3,19 \%)$ & $402,00 €(2,97 \%)$ \\
\hline \multicolumn{4}{|l|}{ Sangrado NMCR } \\
\hline Sangrado NMCR & $560,70 €(4,19 \%)$ & $580,30 €(4,29 \%)$ & $656,30 €(4,85 \%)$ \\
\hline \multicolumn{4}{|l|}{ HPTEC } \\
\hline Costes relacionados con el evento agudo & $105,60 €(0,79 \%)$ & $105,50 €(0,78 \%)$ & $105,50 €(0,78 \%)$ \\
\hline Costes relacionados con el cuidado prolongado & $2.034,40 €(15,21 \%)$ & $2.033,60 €(15,05 \%)$ & $2.034,90 €(15,03 \%)$ \\
\hline \multicolumn{4}{|l|}{ SPT grave } \\
\hline SPT grave & $6.178,50 €(46,20 \%)$ & $6.152,50 €(45,52 \%)$ & $6.169,10 €(45,57 \%)$ \\
\hline COSTE TOTAL & $13.374,70 €$ & $13.516,50 €$ & $13.537,80 €$ \\
\hline
\end{tabular}

AVG: años de vida ganados; AVAC: años de vida ajustados por calidad; EP: embolia pulmonar; HPTEC: hipertensión pulmonar tromboembólica crónica; IC: intracraneal; NMCR: no mayor clínicamente relevante; SPT: síndrome postrombótico; TEV: tromboembolismo venoso; TVP: trombosis venosa profunda

y de $13.537,80 €$ con rivaroxaban. Las partidas de costes que representan una mayor diferencia entre las alternativas de tratamiento comparadas son las relacionadas con el tratamiento anticoagulante (coste farmacológico, administración y monitorización), hemorragias graves y sangrados NMCR. El uso de apixaban respecto al de HBPM/dabigatrán y rivaroxaban supone una reducción de $141,8 €$ y $163,10 €$, con una ganancia de 0,02 y 0,08 AVG y de 0,019 y 0,008 AVAC, respectivamente.

La reducción de eventos clínicos y la consecuente ganancia de AVG y AVAC con apixaban implicarían una mejora tanto de la supervivencia como de la calidad de vida de los pacientes. Con un menor coste por paciente y un incremento en la efectividad, apixaban resultó ser una alternativa de tratamiento dominante respecto a HBPM/dabigatrán y rivaroxaban.

\section{Análisis de sensibilidad}

Los resultados de los ASD univariantes realizados muestran que, tanto en los escenarios en los que la duración del tratamiento considerada varió de seis meses a tres, 12 y durante toda la vida del paciente, como en los que el horizonte temporal evaluado dejó de ser toda la vida del paciente y se redujo a uno, cinco y 10 años, apixaban fue una alternativa de tratamiento dominante respecto a las otras dos alternativas de tratamiento consideradas.

Los resultados del ASP mostraron que en el $98,76 \%$ de las simulaciones apixaban fue una opción de tratamiento do- minante respecto a HBPM/dabigatrán, y en el $88,9 \%$ de las realizadas comparándose con rivaroxaban (Fig. 2).

\section{Discusión}

El TEV se presenta como un sustancial problema de salud pública, puesto que cada año afecta a varios millones de personas en el mundo. Se estima que unas 350.000 personas presentan esta patología cada año en Estados Unidos y una gran mayoría requiere tratamiento hospitalario, frecuentemente costoso [7]. Pese a los escasos datos publicados sobre el coste asociado al tratamiento y manejo de los pacientes afectados por TEV, se sabe que constituye un importante problema económico, de forma que en Estados Unidos se estimó un coste para el TEV de unos 1.500 millones de dólares anuales [42]. En nuestro país se estimó un coste anual en la década anterior de 420,7 millones de euros [1], mientras que estudios más recientes estiman que la hospitalización por EP supone un gasto de 20 millones de euros al año [9]. Además, los pacientes que sobreviven pueden presentar episodios crónicos que requieren hospitalización y tratamientos adicionales. Muchos individuos pueden ver reducida su capacidad laboral (menos horas de trabajo, bajas periódicas, descenso de productividad, etc.), con la consiguiente repercusión sociosanitaria y económica [7]. En definitiva, el TEV, por su elevada prevalencia en la población, es una enfermedad que ocasiona un gasto importante a los sistemas sani- 
Figura 2 Análisis

coste-efectividad de apixaban frente a heparina de bajo peso molecular/dabigatrán $(\mathrm{A})$ y frente a rivaroxaban $(\mathrm{B})$

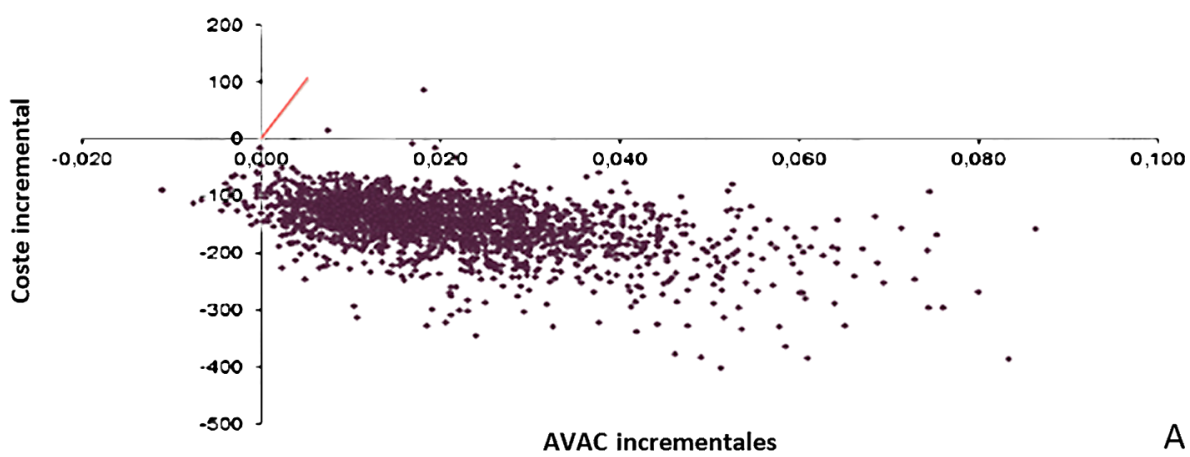

A

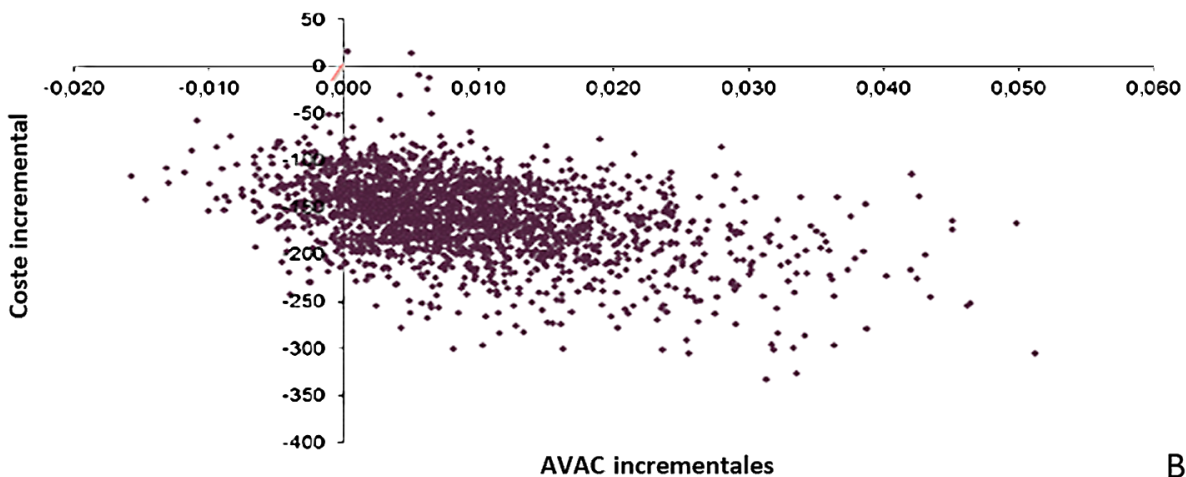

AVAC: años de vida ajustados por calidad tarios. Como consecuencia, disponer de alternativas de tratamiento más eficientes contribuirá a la sostenibilidad del SNS, reduciendo los costes asociados al tratamiento y manejo del TEV e incrementando los beneficios en salud.

Los resultados de este análisis económico ponen de manifiesto un menor número de eventos clínicos en los pacientes tratados con apixaban respecto a las otras dos alternativas de tratamiento evaluadas. Además, la disminución en el número de eventos clínicos en los pacientes tratados con apixaban da lugar a AVG y AVAC adicionales. Adicionalmente, el presente estudio concluye también que el tratamiento de los pacientes con apixaban reduce costes en comparación con HBPM/dabigatrán y con rivaroxaban en pacientes con TEV. Teniendo en cuenta la reducción en el número de eventos clínicos y el ahorro de costes que supone el tratamiento de los pacientes con apixaban, se puede concluir de los dos escenarios analizados que apixaban es una alternativa de tratamiento dominante respecto a HBPM/dabigatrán y rivaroxaban. Se denominan alternativas terapéuticas dominantes aquellas que aportan mayor efectividad y consiguen además una reducción en los costes del manejo de la enfermedad y de las complicaciones frente a la alternativa comparada. Además, apixaban también resultó ser una opción de tratamiento dominante, no solo en el caso base, sino en la totalidad de los ASD realizados en los dos escenarios.

El presente análisis tiene una serie de fortalezas, como por ejemplo ser el único actualmente publicado en nuestro país que compare apixaban frente a HBPM/dabigatrán y rivaroxaban en el tratamiento del primer evento de TEV y prevención de sus recurrencias. Los resultados de los AS realizados modificando los parámetros que podrían comprometer al modelo corroboraron su robustez puesto que, pese a las numerosas variaciones realizadas, los resultados mostraron que el tratamiento con apixaban vs HBPM/dabigatrán y $v s$ rivaroxaban es una opción de tratamiento dominante en todos los ASD realizados, es decir, implica una mayor eficacia con una disminución del coste incremental. En la reciente publicación del análisis coste-efectividad que utilizó el mismo modelo que el descrito en el presente trabajo, apixaban resultó también una alternativa terapéutica dominante frente al tratamiento con HBPM/AVK en el tratamiento del TEV y prevención de recurrencias, tanto en el análisis del caso base como en los ASD [13]. En el ASP este estudio mostró dominio de apixaban en el $89 \%$ de las simulaciones.

Este estudio no está exento de limitaciones, que se deben tener en cuenta a la hora de interpretar los resultados. Entre ellas cabe destacar el hecho de haber empleado datos de ensayos clínicos en vez de datos de estudios que reflejen la práctica médica diaria (datos de vida real que reflejan efectividad en vez de eficacia), pero estos son los datos disponibles en el momento de la comercialización de un nuevo medicamento; otra posible limitación es el hecho de que los resultados en salud derivados de emplear los fármacos evaluados en este estudio se han obtenido de comparaciones indirectas y metanálisis en red, en vez de comparaciones directas entre los tres medicamentos. 
Otra limitación de esta evaluación económica es el hecho de que los valores de utilidad empleados no corresponden a la población española, dado que no localizaron datos específicamente referidos a pacientes en España. Sin embargo, los datos de utilidad incluidos en este modelo fueron validados por el panel de expertos, considerándose de esta forma que eran valores representativos de nuestro país, al no disponer de datos directos de pacientes en nuestro país.

A pesar de las limitaciones descritas, el presente estudio de evaluación económica podría ayudar a facilitar la toma de decisiones a la hora de prescribir un determinado tratamiento en la práctica clínica, dado que el tratamiento con apixaban es una terapia dominante que origina menos costes al SNS y más resultados en salud para el paciente, en comparación con HBPM/dabigatrán y rivaroxaban.

Fuente de financiación El proyecto ha sido financiado con una beca no condicionada de Bristol-Myers Squibb S.A.U. y Pfizer S.L.U.

Conflicto de intereses FdA, IE e IO desarrollan su actividad profesional en Pharmacoeconomics \& Outcomes Research Iberia (PORIB), una consultora especializada en evaluación económica de tecnologías sanitarias que ha recibido remuneración de Bristol-Myers Squibb S.A.U. para la realización de este proyecto.

LAS y FGB han recibido honorarios de PORIB por labores de asesoría en relación con el desarrollo del presente proyecto. AN declara no tener conflicto de intereses.

PG desarrolla su actividad profesional en Bristol-Myers Squibb. JS desarrolla su actividad profesional en Pfizer S.L.U.

Los autores declaran que el soporte económico no ha interferido en el desarrollo del trabajo.

Open Access This article is distributed under the terms of the Creative Commons Attribution-NonCommercial 4.0 International License (http://creativecommons.org/licenses/by-nc/4.0/), which permits any noncommercial use, distribution, and reproduction in any medium, provided you give appropriate credit to the original author(s) and the source, provide a link to the Creative Commons license, and indicate if changes were made.

\section{Bibliografía}

1. Botella G. Reflexiones sobre la enfermedad tromboembólica venosa. An Med Interna. 2003;20:447-50.

2. Goldhaber SZ, Bounameaux H. Pulmonary embolism and deep vein thrombosis. Lancet. 2012;379(9828):1835-46.

3. Cohen AT, Agnelli G, Anderson FA, et al. VTE Impact Assessment Group in Europe (VITAE). Venous thromboembolism (VTE) in Europe. The number of VTE events and associated morbidity and mortality. Thromb Haemost. 2007;98(4):756-64.

4. Laporte S, Mismetti P, Décousus H, et al. RIETE Investigators. Clinical predictors for fatal pulmonary embolism in 15,520 patients with venous thromboembolism: Findings from the Registro Informatizado de la Enfermedad TromboEmbolica venosa (RIETE). Registry Circulation. 2008;117(13):1711-6.

5. Liu X, Phatak H, Dillon R, Mitchell S. Epidemiology and mortality of venous thromboembolism across patient populations: A systematic literature review. Value Health. 2013;16:A1-298.

6. Guijarro Merino R, Montes Santiago J, San Román Terán CM. Epidemiología hospitalaria de la enfermedad tromboembólica venosa en España. Med Clin (Barc). 2008;131(Suppl 2):2-9.
7. Páramo JA, Lecumberri R. Enfermedad tromboembólica venosa: una llamada urgente a la acción. Med Clin (Barc). 2009;133(14):547-51.

8. Wells P, Anderson D. The diagnosis and treatment of venous thromboembolism. Hematology Am Soc Hematol Educ Program. 2013;2013:457-63.

9. Grupo Multidisciplinario para el Estudio de la Enfermedad Tromboembólica en España. Estudio sobre la enfermedad tromboembólica venosa en España [Internet]. Sociedad Española de Medicina Interna: Madrid, 2006 [citado 1 Ago 2014]. Disponible en: https://www.fesemi.org/sites/default/files/documentos/ publicaciones/estudio-etv.pdf

10. Konstantinides S, Torbicki A, Agnelli G, et al. ESC Guidelines on the Diagnosis and Management of Acute Pulmonary Embolism. Rev Esp Cardiol. 2015;68(1):64.

11. Caro JJ, Briggs AH, Siebert U, Kuntz KM, Force I-SMGRPT. Modeling good research practices-overview: A report of the ISPORSMDM Modeling Good Research Practices Task Force-1. Value Health. 2012;15(6):796-803.

12. Siebert U, Alagoz O, Bayoumi AM, et al. State-transition modeling: a report of the ISPOR-SMDM Modeling Good Research Practices Task Force-3. Value Health. 2012;15(6):812-20.

13. Elías I, Oyagüez I, Alvarez-Sala LA, et al. Cost-effectiveness analysis of apixaban compared to low-molecular weight heparins and vitamin $\mathrm{k}$ antagonists for treatment and secondary prevention of venous thromboembolism. Farm Hosp. 2016;40(03):187-208.

14. National Institute for Health and Care Excellence (NICE), Final appraisal determination-Rivaroxaban for treating pulmonary embolism and preventing recurrent venous thromboembolism, Issue date: April 2013, ID569. Disponible en: http://guidance.nice.org.uk/TA/Wave22/20/FAD/FinalAppraisal Determination/pdf/English

15. National Institute for Health and Care Excellence (NICE), Final appraisal determination-Final appraisal determinationRivaroxaban for the treatment of deep vein thrombosis and prevention of deep vein thrombosis and pulmonary embolism, Issue date: May 2013, ID437. Disponible en: http://guidance.nice.org.uk/TA/Wave22/17/FAD/FinalAppraisal Determination/pdf/English

16. Kang N, Sobieraj DM. Indirect treatment comparison of new oral anticoagulants for the treatment of acute venous thromboembolism. Thromb Res. 2014;133(6):1145-51.

17. Cohen AT, Hamilton M, Mitchell SA, et al. Comparison of the novel oral anticoagulants apixaban, dabigatran, edoxaban, and rivaroxaban in the initial and long-term treatment and prevention of venous thromboembolism. Systematic review and network metaanalysis PLoS One. 2015;10(12):e0144856.

18. Poli D, Grifoni E, Antonucci E, et al. Incidence of recurrent venous thromboembolism and of chronic thromboembolic pulmonary hypertension in patients after a first episode of pulmonary embolism. J Thromb Thrombolysis. 2010;30(3):294-9.

19. Prandoni P, Noventa F, Ghirarduzzi A, et al. The risk of recurrent venous thromboembolism after discontinuing anticoagulation in patients with acute proximal deep vein thrombosis or pulmonary embolism. A prospective cohort study in 1,626 patients. Haematologica. 2007;92(2):199-205.

20. Agnelli G, Buller HR, Cohen A, et al. AMPLIFY-EXT Investigators. Apixaban for extended treatment of venous thromboembolism. N Engl J Med. 2013;368(8):699-708.

21. Miniati M, Monti S, Bottai M, et al. Survival and restoration of pulmonary perfusion in a long-term follow-up of patients after acute pulmonary embolism. Medicine. 2006;85(5):253-62.

22. Prandoni $P$, Villalta $S$, Bagatella $P$, et al. The clinical course of deep-vein thrombosis. Prospective long-term follow-up of 528 symptomatic patients. Haematologica. 1997;82(4):423-8. 
23. Linkins L, O'Donnell M, Julian JA, Kearon C. Intracranial and fatal bleeding according to indication for long-term oral anticoagulant therapy. J Thromb Haemost. 2010;8(10):2201-7.

24. Ariesen M, Claus S, Rinkel G, Algra A. Risk factors for intracerebral hemorrhage in the general population: A systematic review. Stroke. 2003;34:2060-5.

25. Agnelli G, Buller HR, Cohen A, et al. AMPLIFY Investigators. Oral apixaban for the treatment of acute venous thromboembolism. N Engl J Med. 2013;369(9):799-808.

26. Kind P, Dolan P, Gudex C, Williams A. Variations in population health status: results from a United Kingdom national questionnaire survey. BMJ. 1998;316(7133):736-41.

27. Locadia M, Bossuyt PM, Stalmeier PF, et al. Treatment of venous thromboembolism with vitamin $\mathrm{K}$ antagonists: Patients' health state valuations and treatment preferences. Thromb Haemost. 2004;92(6):1336-41.

28. Ghofrani HA, D'Armini AM, Grimminger F, et al. Riociguat for the treatment of chronic thromboembolic pulmonary hypertension. N Engl J Med. 2013;369(4):319-29.

29. National Institute of Health and Care Excellence (NICE), Venous thromboembolism: Reducing the risk, January 2010, NICE CG92.

30. Hogg $\mathrm{K}$, Kimpton $\mathbf{M}$, Carrier $\mathrm{M}$, et al. Estimating quality of life in acute venous thrombosis. JAMA Intern Med. 2013;173(12):1067-72.

31. Sullivan PW, Slejko JF, Sculpher MJ, Ghushchyan V. Catalogue of EQ-5D scores for the United Kingdom. Med Decis Making. 2011;31(6):800-80.

32. Lenert LA, Soetikno RM. Automated computer interviews to elicit utilities: Potential applications in the treatment of deep venous thrombosis. J Am Med Inform Assoc. 1997;4(1):49-56.

33. Gage BF, Cardinalli AB, Owens DK. The effect of stroke and stroke prophylaxis with aspirin or warfarin on quality of life. Arch Intern Med. 1996;156(16):1829-36.

34. Base de Datos de Medicamentos del Consejo General de Colegios Farmacéuticos [consultado 31 Jul 2014]. Disponible en: https://botplusweb.portalfarma.com/botplus.aspx
35. Ministerio de Sanidad, Servicios Sociales e Igualdad. Relación informativa de medicamentos afectados por las deducciones establecidas en el Real Decreto Ley 8/2010 de 20 de mayo por el que se adoptan medidas extraordinarias para la reducción del déficit público. [Internet]. Madrid: Ministerio de Sanidad, Servicios Sociales e Igualdad, 2010 [actualizado Jul 2015, citado 31 Jul 2015]. Disponible en: http://www. msssi.gob.es/profesionales/farmacia/pdf/deduccionesJul2014.pdf

36. Oblikue Consulting. Base de datos de costes sanitarios eSalud [Internet]. Barcelona: Oblikue Consulting, 2014 [citado 1 Ago 2014]. Disponible en: http://www.oblikue.com/bddcostes/

37. Tarifas según Grupo Relacionado por Diagnóstico. Boletines Oficiales de las Comunidades Autónomas. En: Oblikue Consulting. Base de datos de costes sanitarios eSalud [Internet]. Barcelona: Oblikue Consulting, 2015 [citado 1 Ago 2014]. Disponible en: http://www.oblikue.com/bddcostes/

38. Ministerio de Sanidad, Servicios Sociales e Igualdad, Instituto de información sanitaria. Registro de altas. CIE9 MC-CMBD 2011. En: Portal estadístico del Ministerio de Sanidad, Servicios Sociales e Igualdad [Internet]. Madrid: Ministerio de Sanidad, Servicios Sociales e Igualdad, 2013 [citado 1 Ago 2014]. Disponible en: http://pestadistico.msc.es

39. Monreal M, González-Rojas N, Vieta A, Wolowacz SE. Análisis económico de dabigatrán etexilato en prevención primaria del tromboembolismo venoso tras artroplastia total de cadera o rodi1la. Pharmacoecon Span Res Artic. 2009;6(4):105-62.

40. Ministerio de Sanidad, Servicios Sociales e Igualdad. Instituto de Información Sanitaria (2013). Registro de altas GRD 174-CMBD 2011.

41. Ministerio de Sanidad, Servicios Sociales e Igualdad. Instituto de Información Sanitaria (2013). Registro de altas GRD 123-CMBD 2011.

42. Montes J, González L, Amador L, et al. Tratamiento domiciliario de la trombosis venosa profunda. Comparación de costes con la hospitalización convencional. An Med Interna (Madrid). 2005;22:369-72. 